\title{
Desempenho de reatores anaeróbios operados em bateladas seqüenciais (escala piloto) no tratamento de esgoto sanitário
}

\section{Performance of anaerobic sequencing batch reactors (pilot-scale) in domestic sewage treatment}

\author{
Arnaldo Sarti ${ }^{1}$; Marcelo Zaiat ${ }^{2}$; Eugenio Foresti ${ }^{3}$
}

Resumo

\begin{abstract}
Este trabalho apresenta os resultados obtidos durante 70 dias na operação de quatro reatores anaeróbios operados sob a forma de bateladas seqüenciais em escala piloto, para tratamento de esgotos sanitários do Campus da USP de São Carlos. Com 1,2 $\mathrm{m}^{3}$ de volume total, cada reator foi concebido para o tratamento de $1,95 \mathrm{~m}^{3} \cdot \mathrm{d}^{-1}$ de esgoto sanitário, com concepções geométricas (relação H-altura/D-diâmetro), mecânicas (agitação mecânica ou recirculação de líquido) e tipo de retenção de biomassa diferentes. Três unidades eram reatores anaeróbios operados em bateladas seqüenciais (ASBR-“Anaerobic Sequencing Batch Reactor") do tipo convencional com biomassa granular e outra do tipo reator anaeróbio operado em bateladas seqüenciais contendo biomassa imobilizada em espuma de poliuretano (ASBBR -“Anaerobic Sequencing Batch Biofilm Reactor"). Os reatores foram inoculados e o ciclo total (batelada) tinha duração de oito horas. O monitoramento dos reatores incluiu a determinação de demanda química de oxigênio (DQO), pH, sólidos suspensos totais ( $\mathrm{SST}$ ) e sólidos suspensos voláteis, ácidos voláteis totais, alcalinidade a bicarbonato e a concentração de metano no biogás. Os dois reatores ASBR com recirculação de líquido não apresentaram desempenho satisfatório. Os valores médios de eficiência de remoção de DQO e de SST foram próximos de $40 \%$ e $60 \%$, respectivamente. Nos reatores, ASBR com agitação mecânica e, ASBBR com biomassa imobilizada, os resultados médios obtidos foram melhores. O reator ASBBR atingiu eficiência média de $61 \%$ e $75 \%$ em remoção de DQO e SST, enquanto no ASBR com agitação mecânica chegou-se a $60 \%$ e $79 \%$, respectivamente.
\end{abstract}

Palavras-chave: Tratamento anaeróbio. Reator anaeróbio. Bateladas seqüenciais. Esgoto sanitário.

\begin{abstract}
This study shows the results obtained during 70 days using four pilot scale anaerobic sequencing batch reactors, for sewage system treatment of the campus of the University of São Paulo in São Carlos - SP. Each reactor system with $1.2 \mathrm{~m}^{3}$ of total volume, was designed for the treatment of $1.95 \mathrm{~m}^{3} \mathrm{~d}^{3}$ of domestic sewage, with geometrical conceptions (ratio H-height/D-diameter), mechanical conceptions (mechanical mixing or liquid re-circulation) and different biomass retention type. Three of them were ASBR (Anaerobic
\end{abstract}

\footnotetext{
1 Doutor em Engenharia Civil, Pós-Doutor do Departamento de Hidráulica e Saneamento, Laboratório de Processos Biológicos, Escola de Engenharia de São Carlos (USP/ São Carlos). E-mail: arnaldosarti@gmail.com

2 Doutor em Engenharia Civil, Professor do Departamento de Hidráulica e Saneamento, Escola de Engenharia de São Carlos (USP/ São Carlos).

3 Doutor em Engenharia Civil, Professor Titular do Departamento de Hidráulica e Saneamento, Escola de Engenharia de São Carlos (USP/ São Carlos).
} 
Sequencing Batch Reactor) with granular biomass and another one was an ASBBR (Anaerobic Sequencing Batch Biofilm Reactor) with biomass consisting of cubic matrices of polyurethane foam. The reactors were inoculated and operated within an 8-hour batch cycle. The reactors monitoring included the oxygen chemical demand (OCD), $\mathrm{pH}$, total suspended solids (TSS) and volatile suspended solids, volatile fatty acids, alkalinity to bicarbonate and methane concentration. Both ASBR reactors with liquid re-circulation did not show a satisfactory performance. The average values of OCD and TSS removal were close to $40 \%$ and $60 \%$ respectively. In the ASBR reactors with mechanical mixing and ASBBR with immobilized biomass, showed better results. The ASBBR reactor reached average efficiency of $61 \%$ and $75 \%$ in OCD and TSS removal, while in the ASBR with mechanical mixing, it reached $60 \%$ and $79 \%$, respectively.

Key words: Anaerobic treatment. Anaerobic sequencing. Batch reactor. Domestic sewage.

\section{Introdução}

O conhecimento e o interesse pelo tratamento anaeróbio cresceram consideravelmente, nas últimas três décadas, devido ao aumento significativo do número de alternativas, tanto no que se refere à concepção física das unidades de tratamento para conversões biológicas, como na otimização do processo em si. Tem-se conhecimento, hoje, de que os reatores anaeróbios são capazes de tratar grande diversidade de águas residuárias, inclusive de origem doméstica.

O conhecimento adquirido é, sem dúvida, desproporcional ao nível de qualidade de muitos projetos e sistemas implantados. Pode-se afirmar que, no Brasil, a implantação de sistemas mal concebidos e construídos pode ter comprometido, de forma prematura, o avanço dessa tecnologia para tratamento de esgotos sanitários. É evidente que, dentre outros, a disseminação restrita dos conhecimentos pode ser considerada um dos principais fatores responsáveis pelo uso indevido ou má aceitação da tecnologia anaeróbia. É importante que se mencione que os critérios de projeto dos reatores anaeróbios são, ainda, empíricos, o que torna o projeto das unidades de tratamento aparentemente simples. No entanto, a simplicidade do projeto não decorre da simplicidade dos processos, pelo contrário, os processos são muito complexos e o sucesso depende do fornecimento de condições ambientais, nos reatores, que permitam o estabelecimento de mecanismos de autocontrole (OLIVA, 1997).
O incremento da aplicação do tratamento anaeróbio no Brasil pode significar a consagração de sistemas simplificados que, certamente, possuem potencialidade de beneficiar comunidades desprovidas de possibilidade de implementação de soluções mais sofisticadas. Essas unidades simplificadas devem buscar resolver o problema da estabilização da matéria orgânica do esgoto sanitário, de maneira confiável. Os novos reatores anaeróbios com biomassa imobilizada vêm sendo utilizados no tratamento de águas residuárias, e permitem obter bom desempenho com grande estabilidade do processo, devido à capacidade de trabalhar com altos tempos de retenção celular, quando operados com baixos tempos de detenção hidráulica. Esses reatores permitem a imobilização e retenção dos microrganismos na forma de grânulos, flocos e sistemas de biofilmes aderidos a suportes inertes (HENZE et al., 1997).

Para tratamento anaeróbio de esgoto sanitário, os principais tipos de reatores que estão sendo utilizados, na prática, no Brasil são: decanto-digestores (fossas sépticas), lagoas anaeróbias, filtros anaeróbios, reatores anaeróbios de manta de lodo (UASB) e, mais recentemente, os reatores anaeróbios compartimentados e de leito expandido ou fluidificado. Esses reatores podem se apresentar individualmente ou em sistemas combinados, para atender a diversas situações de arranjos de unidades em Estações de Tratamento.

Como alternativa aos reatores anaeróbios de fluxo contínuo, o reator anaeróbio operado em bateladas 
seqüenciais (ASBR) vem recebendo atenção maior nos últimos anos, com pesquisas que visam sua aplicação prática no tratamento de águas residuárias em geral. O tratamento em si é efetuado na mesma unidade, em uma seqüência operacional que compreende as fases: alimentação; tratamento ou reação. Durante essa seqüência, o substrato orgânico é biodegradado na presença de microrganismos anaeróbios, e ocorre a sedimentação da biomassa (lodo) e descarga ou descarte. A operação completa (ciclo total) do ASBR pode ser vista na Figura 1.

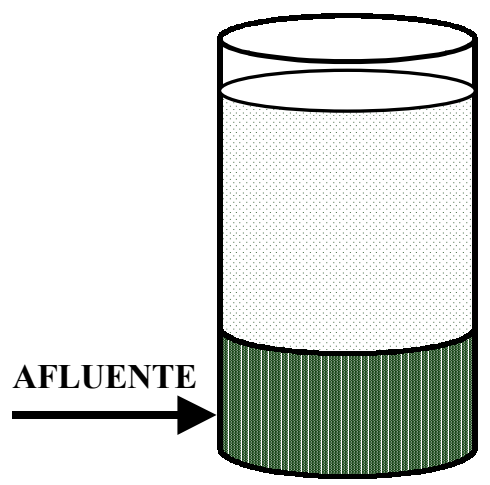

ALIMENTAÇÃO

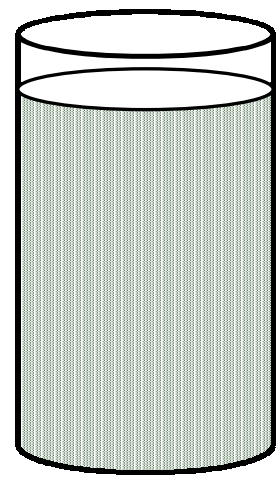

REAÇÃO*

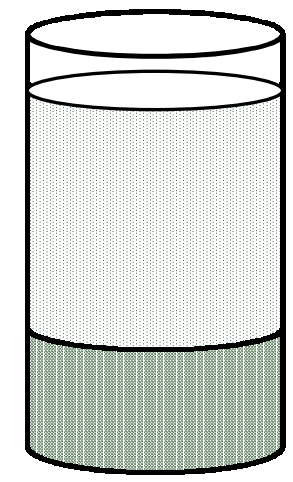

SEDIMENTAÇÃO

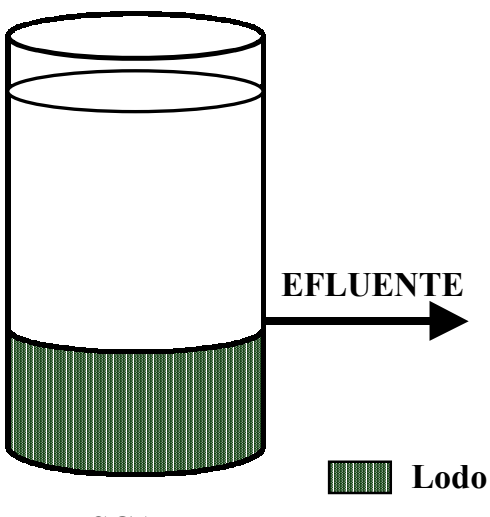

DESCARTE

Figura 1: Etapas do ciclo total de operação do reator operado em bateladas seqüenciais.

O estudo e o desenvolvimento desse tipo de reator anaeróbio com fluxo intermitente são recentes e os resultados obtidos, até o momento, indicam grande potencialidade destas unidades serem utilizadas no tratamento anaeróbio de diversos tipos de substratos. Sua aplicação em maior escala no tratamento de águas residuárias pode ser justificada em casos especiais. Por exemplo, esgoto sanitário de pequenas comunidades, indústrias que lançam efluentes líquidos de forma descontínua ou atividades que geram efluentes apenas em algumas épocas do mês ou ano.

Essa configuração de reator vem sendo estudado de forma efetiva, desde 1991, por grupos de pesquisadores da Universidade Estadual de Iowa (EUA) e da Universidade de Ottawa (Canadá) em escala de laboratório e, no Brasil, por grupos da Escola de Engenharia de São Carlos (EESC/USP) e da Escola de Engenharia do Instituto Mauá de Tecnologia (EEM/IMT). Nessas instituições brasileiras, os projetos de pesquisa têm enfoque no estudo de otimização do reator tipo ASBR e de novas propostas de configurações de reatores (ASBBR), para que a aplicação do sistema operado em bateladas seqüenciais em escala plena se torne viável. No presente trabalho foram projetadas e operadas diferentes configurações de reatores anaeróbios do tipo ASBR em escala piloto, visando à sua aplicabilidade no tratamento de esgoto sanitário.

\section{Material e Métodos}

Existe, na literatura, poucos dados específicos, na literatura, para projetos de reatores anaeróbios operados em bateladas seqüenciais. Por esse motivo, foi necessário adotar alguns parâmetros, para viabilizar o projeto das unidades em escala piloto, já que, na maioria dos trabalhos, os reatores são de escala de laboratório tratando substratos simples e que praticamente, não retratam a realidade de sistemas em maior escala.

Nesse contexto, o projeto dos reatores anaeróbios operados em bateladas foi concebido para tratamento 
de $5,85 \mathrm{~m}^{3} \cdot \mathrm{d}^{-1}$ de esgotos sanitários, coletados da rede que atravessa o Campus da Universidade de São Paulo (São Carlos-SP). As quatro unidades foram projetadas para tratamento de $1,95 \mathrm{~m}^{3} \cdot \mathrm{d}^{-1}$, individualmente, com concepções geométricas, mecânicas (tipo de agitação) e tipo de retenção de biomassa diferentes.

O tempo de ciclo total foi adotado de 8 horas, para todos os reatores, e foi definido com base no trabalho de Ratusznei et al. (2000). A adoção desse tempo de ciclo total implica a realização de três ciclos completos por dia, sendo cada ciclo correspondente aos tempos de enchimento, reação, sedimentação, descarga e repouso para os reatores ASBR. No caso do ASBBR, foi dispensada a fase de sedimentação, por se tratar de reator que contém biomassa imobilizada em meio suporte. As concepções dos reatores anaeróbios envolveram diferentes tipos de configurações como:

\section{Reator Anaeróbio Operado em Bateladas Seqüenciais (ASBR1, ASBR2 e ASBR3)}

Na configuração do tipo ASBR foram projetadas três unidades, cada uma com volume total de 1,20 $\mathrm{m}^{3}$, tendo diferentes tipos de agitação e característica geométrica por meio da relação altura $(\mathrm{H}) /$ diâmetro(D). Dois reatores ASBR tinham como forma de agitação a recirculação de líquido por bomba centrífuga (Jacuzzi - modelo 5JL15), mas com relações H/D distintas, ao passo que o outro possuía agitação mecânica de líquido, mas relação H/D igual a um dos ASBR concebidos com recirculação de líquido. Nesses reatores ASBR, destinou-se 1/3 do volume total como o volume para acumulação de biomassa ou lodo biológico $\left(0,4 \mathrm{~m}^{3}\right)$, enquanto o volume para o biogás gerado foi adotado como sendo de $12 \%$ do volume total. Todas as unidades do tipo ASBR possuíam o mesmo volume líquido removível, ou seja, a ser tratado a cada ciclo de $0,65 \mathrm{~m}^{3}$.

\section{Reator Anaeróbio operado em Batelada Seqüencial com Biomassa Imobilizada (ASBBR)}

Para a concepção do ASBBR com biomassa imobilizada em suporte inerte, baseou-se no trabalho de Ratusznei et al. (2000). Tal configuração do tipo ASBBR foi projetada com mesmo volume total $(1,20$ $\mathrm{m}^{3}$ ), e consequentemente, igual volume líquido removível a cada ciclo $\left(0,65 \mathrm{~m}^{3}\right)$ dos ASBR.

Esta configuração ASBBR difere pela presença, no interior da unidade, de suportes inertes para imobilização da biomassa (lodo). A unidade tinha como forma de agitação, o meio mecânico, e que as biopartículas ficavam confinadas em uma espécie de "cesto cilíndrico" de tela de inox 316, com furos de $15 \mathrm{~mm}$ de diâmetro, ao redor do eixo de agitação. O volume para o suporte ou volume para acomodação do material suporte inerte no cesto cilíndrico foi o mesmo destinado para acumulação de lodo dos ASBRs. Para o preenchimento do cesto foram empregados cubos de espuma de poliuretano $(50 \mathrm{~mm}$ de aresta) com densidade de $23 \mathrm{~kg} / \mathrm{m}^{3}$.

Salienta-se que, abaixo do cesto e junto ao fundo do reator, destinou-se certo volume para descarte eventual de lodo biológico $\left(0,075 \mathrm{~m}^{3}\right)$. O mesmo valor foi adotado para o volume de biogás na parte superior do reator.

As principais características dimensionais e operacionais dos reatores são apresentadas nas Tabelas 1 e 2, enquanto na Tabela 3 visualizam-se os tempos das etapas envolvidas na execução do ciclo total dos reatores. No caso dos reatores ASBR, cada ciclo total correspondeu aos tempos de enchimento, reação, sedimentação, descarga e repouso. No ASBBR, foi dispensada a fase de sedimentação por se tratar de reator contendo biomassa imobilizada em meio suporte. 
Tabela 1. Características dimensionais e operacionais dos reatores ASBR

\begin{tabular}{lccc}
\hline Reator & ASBR1 & ASBR2 & ASBR3 \\
\hline Material & Polietileno & Polietileno & Fibra de vidro \\
Altura (H) & $1500 \mathrm{~mm}$ & $1500 \mathrm{~mm}$ & $2400 \mathrm{~mm}$ \\
Diâmetro (D) & $1000 \mathrm{~mm}$ & $1000 \mathrm{~mm}$ & $800 \mathrm{~mm}$ \\
Relação (H/D) & 1,5 & 1,5 & 3,0 \\
Volume p/ Biogás $\left(\mathrm{V}_{\mathrm{B}}\right)$ & $0,15 \mathrm{~m}^{3}$ & $0,15 \mathrm{~m}^{3}$ & $0,15 \mathrm{~m}^{3}$ \\
Volume Líquido Removível $\left(\mathrm{V}_{\mathrm{L}}\right)$ & $0,65 \mathrm{~m}^{3}$ & $0,65 \mathrm{~m}^{3}$ & $0,65 \mathrm{~m}^{3}$ \\
Volume p/ Lodo $\left(\mathrm{V}_{\mathrm{S}}\right)$ & $0,40 \mathrm{~m}^{3}$ & $0,40 \mathrm{~m}^{3}$ & $0,40 \mathrm{~m}^{3}$ \\
Volume Útil $\left(\mathrm{V}_{\mathrm{L}}+\mathrm{V}_{\mathrm{S}}\right)$ & $1,05 \mathrm{~m}^{3}$ & $1,05 \mathrm{~m}^{3}$ & $1,05 \mathrm{~m}^{3}$ \\
Volume Total $\left(\mathrm{V}_{\mathrm{B}}+\mathrm{V}_{\mathrm{L}}+\mathrm{V}_{\mathrm{S}}\right)$ & $1,20 \mathrm{~m}^{3}$ & $1,20 \mathrm{~m}^{3}$ & $1,20 \mathrm{~m}^{3}$ \\
Agitação (etapa de reação) & Recirculação (líquido) & Mecânica (30 rpm) & Recirculação (líquido) \\
Impelidores (2) & - & Turbina (6 pás planas) & - \\
Vazão de recirculação & $1,5 \mathrm{~m}^{3} \cdot \mathrm{h}^{-1}$ & - & $1,5 \mathrm{~m}^{3} \cdot \mathrm{h}^{-1}$ \\
Forma de agitação & $* *$ Intermitente & Contínua & $* *$ Intermitente \\
\hline
\end{tabular}

*Volume tratado por ciclo, ** Recirculação acionada por 10 minutos a cada 1 hora na fase de reação

Tabela 2. Características dimensionais e operacionais do reator ASBBR

\begin{tabular}{cc}
\hline Reator & ASBBR \\
\hline Material & Polietileno \\
Altura $(\mathrm{H})$ & $1500 \mathrm{~mm}$ \\
Diâmetro $(\mathrm{D})$ & $1000 \mathrm{~mm}$ \\
Relação $(\mathrm{H} / \mathrm{D})$ & 1,5 \\
Volume p/ Biogás $\left(\mathrm{V}_{\mathrm{B}}\right)$ & $0,075 \mathrm{~m}^{3}$ \\
*Volume Líquido Removível $\left(\mathrm{V}_{\mathrm{L}}\right)$ & $0,65 \mathrm{~m}^{3}$ \\
Volume p/ Descarte Lodo $\left(\mathrm{V}_{\mathrm{D}}\right)$ & $0,075 \mathrm{~m}^{3}$ \\
**Volume p/ Suporte $\left(\mathrm{V}_{\mathrm{P}}\right)$ & $0,40 \mathrm{~m}^{3}$ \\
Volume Útil ( $\left.\mathrm{V}_{\mathrm{L}}+\mathrm{V}_{\mathrm{P}}\right)$ & $1,05 \mathrm{~m}^{3}$ \\
Volume Total ( $\left.\mathrm{V}_{\mathrm{B}}+\mathrm{V}_{\mathrm{L}}+\mathrm{V}_{\mathrm{P}}+\mathrm{V}_{\mathrm{D}}\right)$ & $1,20 \mathrm{~m}^{3}$ \\
Agitação (contínua-etapa reação) & Mecânica (40 rpm) \\
Impelidores $(2)$ & Turbina $(6$ pás planas) \\
\hline
\end{tabular}

*Volume tratado por ciclo; ** Volume p/ acomodação do suporte inerte

Tabela 3. Etapas de operação dos reatores para ciclo total de 8 horas.

\begin{tabular}{cc}
\hline ASBR1/ASBR2/ASBR3 & ASBBR \\
\hline Enchimento: $2 \mathrm{~h}$ & Enchimento: $2 \mathrm{~h}$ \\
Reação com agitação: $3 \mathrm{~h}$ & Reação com agitação: $5 \mathrm{~h}$ \\
Sedimentação: $2 \mathrm{~h}$ & Não há etapa de Sedimentação \\
Descarga: $0,5 \mathrm{~h}$ & Descarga: $0,5 \mathrm{~h}$ \\
Repouso: $0,5 \mathrm{~h}$ & Repouso: $0,5 \mathrm{~h}$ \\
\hline
\end{tabular}

Pela complexidade de operação simultânea das diferentes configurações de reatores, concebeu-se o sistema de automação, composto de válvulas automatizadas para abertura e fechamento das tubulações (entrada e saída) dos reatores; motores dos agitadores; bombas de recirculação; e bomba de esgoto bruto para alimentação dos sistemas. Todos esses dispositivos foram interligados a um microcomputador para permitir seus acionamentos automáticos. Os motores dos agitadores dos reatores ASBR2 e ASBBR, apenas o acionamento foi mantido ao microcomputador. A velocidade de agitação foi controlada com o uso de regulador eletrônico (Tipo PWM), sendo o ajuste realizado por meio de medidas fornecidas por tacômetros (rpm) instalados nos eixos dos referidos reatores. Na Figura 2 visualiza-se o Fluxograma esquemático proposto de operação simultânea dos reatores. 


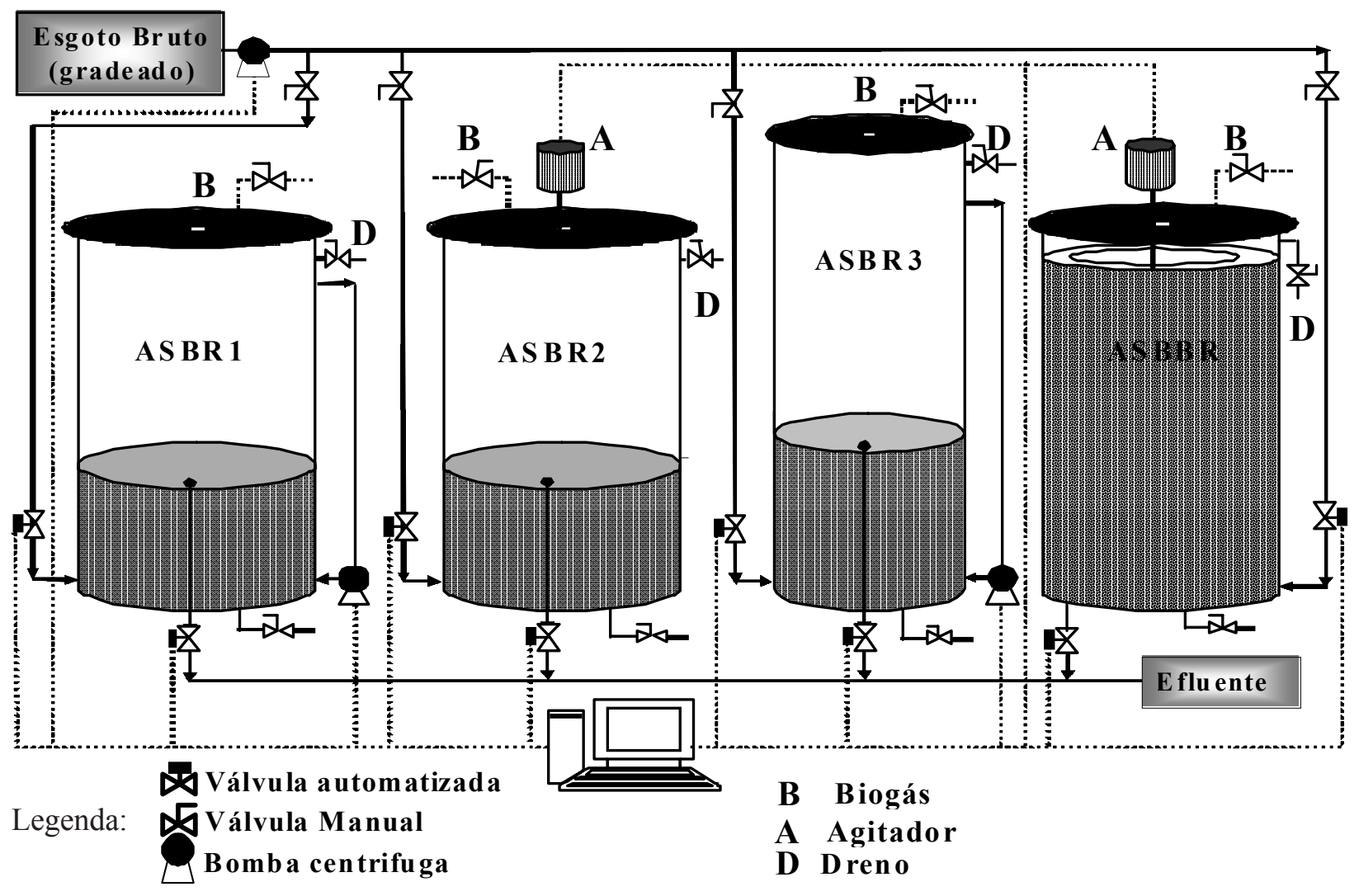

Figura 2. Fluxograma proposto de operação simultânea dos reatores.

As válvulas automatizadas foram montadas com válvulas de PVC $\left(\phi 1^{1 / 2 "}\right)$ vendidas comercialmente e moto-redutor acoplado para maior rapidez e segurança de fechamento. A essas válvulas foram acopladas chaves de "fim de curso" a fim de limitarse o ângulo de abertura das válvulas. Essas válvulas foram acopladas nas tubulações de entrada e saída dos reatores com diâmetro de 1 ".

Nos reatores ASBR1 e ASBR3, com recirculação por bomba, foram instalados pressostatos que tinham a função de sensores de nível e, também de proteção, para que as bombas não acionassem com volume inferior no reator a $1,0 \mathrm{~m}^{3}$, no caso de eventual falta de energia durante a operação de enchimento dos reatores. Nesse sentido, para manter o sistema de automação atuando sobre as válvulas automatizadas, instalou-se sistema "No-Break" para dar prosseguimento às ações agendadas no software, mesmo sem a presença de energia elétrica por período de até 3 horas. Na parte superior dos reatores colocaram-se drenos para não haver transbordamento, bem como tubulação de coleta de gases.
As ações seqüenciais para cada etapa do ciclo total (enchimento, reação, sedimentação, descarga e repouso) foram agendadas, para cada tipo reator (ASBR ou ASBBR), de forma temporal (minutos) por meio de software específico desenvolvido em DELPHI 3 em MS-Windows. Para esta tarefa, empregou-se uma placa de aquisição de dados (entradas e saídas digitais e analógicas) instalada no microcomputador e interligada aos reatores.

A seqüência lógica das ações seqüenciais era empreendida pelo uso do software nas ações de acionamento para a bomba de alimentação de esgoto bruto (gradeado), abertura/fechamento de válvulas automatizadas (enchimento e descarte), bem como as bombas de recirculação e agitadores mecânicos. $\mathrm{Na}$ Tabela 4, visualizam-se as fases do ciclo de operação dos reatores utilizada como base para a estruturação das ações seqüenciais a serem executadas por cada unidade de tratamento na operação em paralelo. 
Tabela 4: Etapas de operação em paralelo no período de 24 horas

\begin{tabular}{l|l|l|l|l|l|l|l|l|l|l|l|l|l|l|l|l|l|l|l|l|l|l|l|l|l}
\hline Reator & $\mathbf{1}$ & $\mathbf{2}$ & $\mathbf{3}$ & $\mathbf{4}$ & $\mathbf{5}$ & $\mathbf{6}$ & $\mathbf{7}$ & $\mathbf{8}$ & $\mathbf{9}$ & $\mathbf{1 0}$ & $\mathbf{1 1}$ & $\mathbf{1 2}$ & $\mathbf{1 3}$ & $\mathbf{1 4}$ & $\mathbf{1 5}$ & $\mathbf{1 6}$ & $\mathbf{1 7}$ & $\mathbf{1 8}$ & $\mathbf{1 9}$ & $\mathbf{2 0}$ & $\mathbf{2 1}$ & $\mathbf{2 2}$ & $\mathbf{2 3}$ & $\mathbf{2 4}$ \\
\hline ASBR1 & E & E & R & R & R & S & S & D & E & E & R & R & R & S & S & D & E & E & R & R & R & S & S & D \\
\hline ASBR2 & E & E & R & R & R & S & S & D & E & E & R & R & R & S & S & D & E & E & R & R & R & S & S & D \\
\hline ASBR3 & E & E & R & R & R & S & S & D & E & E & R & R & R & S & S & D & E & E & R & R & R & S & S & D \\
\hline ASBBR & E & E & R & R & R & R & R & D & E & E & R & R & R & R & R & D & E & E & R & R & R & R & R & D \\
\hline
\end{tabular}

Legenda: $\mathbf{E}$ - enchimento, $\mathbf{R}$ - reação, $\mathbf{S}$ - sedimentação e $\mathbf{D}$ - descarga e repouso.

\section{Análises físico-químicas}

O monitoramento dos reatores ASBR e ASBBR, durante 70 dias, envolveu análises físico-químicas (afluente e efluente) como DQO bruta e filtrada (membrana 1,2 mm), $\mathrm{pH}$, sólidos suspensos totais e voláteis (SSV) segundo métodos descritos pelo American Public Health Association (1995). Ácidos voláteis totais (AVT) como ácido acético e alcalinidade a bicarbonato $(\mathrm{AB})$ foram analisados de acordo com método descrito por Dilallo e Albertson (1961) e modificado por Ripley, Boyle e Converse (1986). Os gases gerados na biodegradação anaeróbia foram monitorados por meio de cromatografia gasosa (Cromatógrafo Gow-Mac com detector de condutividade térmica). Para quantificar a concentração de sólidos totais (ST) e voláteis (SV) no lodo de inóculo seguiu-se metodologia descrita pelo American Public Health Association (1995) e no suporte inerte (espuma de poliuretano) por Ribeiro et al. (2005).

Os reatores foram mantidos em temperatura ambiente durante toda a fase experimental $\left(29 \pm 5^{\circ} \mathrm{C}\right)$. A partir dos resultados obtidos nas referidas análises físico-químicas de monitoramento (20 amostras pontuais), feitas desde a partida dos reatores, podese comparar o desempenho dos sistemas conforme as diferentes formas geométricas, os reatores ASBR1 $(\mathrm{H} / \mathrm{D}=1,5)$ e ASBR3 $(\mathrm{H} / \mathrm{D}=3,0)$ e, também, pelo tipo de agitação, recirculação de líquido e agitação mecânica nas unidades com mesmo $\mathrm{H} / \mathrm{D}=1,5$, os reatores ASBR1 e ASBR2. Conjuntamente, analisouse o desempenho do reator ASBBR com biomassa imobilizada em cubos de espuma de poliuretano, para efeito de comparação com os reatores ASBR.

\section{Inoculação dos reatores}

A princípio, os reatores ASBRs foram inoculados com aproximadamente 4001 de lodo granular (volume para lodo), e o ASBBR, com o mesmo lodo (60 1 de lodo granular desintegrado e $60 l$ de água- total 120 l), sendo este proveniente de um reator UASB que trata águas residuárias de abatedouros de aves (Avícola Dacar-Tiête -SP). No ASBBR, completouse o volume $\left(0,65 \mathrm{~m}^{3}\right)$ com esgoto sanitário e manteve-se a agitação mecânica durante sete dias (sem paradas) permitido a homogeneização para propiciar a aderência de biomassa nos cubos de espuma de poliuretano. A opção por lodo granular decorreu diretamente da melhor característica de sedimentação desse tipo de lodo, verificada por vários autores na literatura no emprego de reatores ASBR, em escala de bancada (SUNG; DAGUE, 1995; WIRTZ; DAGUE, 1996).

\section{Resultados e Discussão}

O inóculo granular utilizado apresentava concentração inicial em termos de ST e SV de 64,1 gST. $l^{-1}$ e 52,8 gSV. $l^{-1}$, respectivamente. Nos ASBR, considerando-se o volume adicionado (400 1), obtevese concentrações de $25,6 \mathrm{kgST}$ e $21,1 \mathrm{kgSV}$ e, levando-se em conta o volume total dos reatores de $1,2 \mathrm{~m}^{3}$, as concentrações de SV e ST na partida foram 
de 21,3 kgST.m ${ }^{-3}$ e 17,6 kgSV.m ${ }^{-3}$, respectivamente. A bibliografia especializada recomenda que as cargas biológicas iniciais, durante a partida de um reator anaeróbio, sejam da ordem de 0,05 a 0,15 $\mathrm{kgDQO} . \mathrm{kgSV}^{-1}$. dia $^{-1}$, dependendo do tipo de efluente que está sendo tratado. Essas cargas devem ser aumentadas gradativamente, em função da eficiência do sistema. A carga biológica, durante o regime dinâmico aparente, pode atingir, de acordo com o tipo de afluente tratado, valores em torno de 2,0 kgDQO.kgSV ${ }^{-1}$. dia ${ }^{-1}$ (CHERNICHARO; VAN HAANDEL; CALVALCANTI, 1999).

Nos reatores ASBR, a carga biológica ou carga de "lodo" foi determinada por meio do produto do volume tratado por dia em cada reator de $1,95 \mathrm{~m}^{3} . \mathrm{d}^{-}$ ${ }^{1}$ e a concentração de substrato mínima $\left(0,35 \mathrm{~kg} \cdot \mathrm{m}^{-}\right.$ $\left.{ }^{3}\right)$ ou máxima $\left(0,80 \mathrm{~kg} \cdot \mathrm{m}^{-3}\right)$ no período de 70 dias. Tal produto dividido pela massa de lodo admitida ao reator $(21,1 \mathrm{kgSV})$ pode-se determinar os valores de carga de lodo de 0,03 a $0,07 \mathrm{kgDQO} . \mathrm{kgSV}^{-1} \cdot \mathrm{d}^{-1}$ Esses dados indicam que os reatores dispuseram de quantidades de lodo maiores do que a necessária para garantir o sucesso da partida. No ASBBR, a quantidade de biomassa $(\mathrm{kg})$ aderida ao suporte de poliuretano em termos de concentração de sólidos totais e voláteis foi crescente durante o ensaio experimental (Figura 3). Os dados eram de 0,28 kgST.kgsuporte ${ }^{-1}(2,5 \mathrm{kgST})$ e $0,21 \mathrm{kgSV} . \mathrm{kgsuporte}^{-}$ ${ }^{1}(1,9 \mathrm{kgSV})$ no $1^{\circ}$ dia de operação, e que, ao final de $70^{\circ}$ dia de operação, alcançou-se concentrações de 0,62 kgST.kgsuporte ${ }^{-1}(5,6 \mathrm{kgST})$ e 0,45 kgSV.kgsuporte ${ }^{-1}(4,0 \mathrm{kgSV})$.

A comparação entre os reatores ASBR1/ASBR2 (diferente tipo de agitação e mesma relação H/D) e o ASBR1/ASBR3 (igual tipo de agitação e diferente relação H/D) notou-se desempenho semelhante das unidades com recirculação de líquido (Tabela 5). A baixa retenção de sólidos foi o principal problema enfrentado, responsável pelo fraco desempenho apresentado, mesmo tendo essas unidades sido inoculadas (Tabela 5). Em virtude de não existir aparato de separação de fases, houve passagem de lodo biológico pela bomba centrífuga, ocasionando sua fragmentação, dificultando a formação e retenção de biomassa adequada, bem como reduzindo sua característica de sedimentação. Hollopeter e Dague (1994) e Ndon e Dague (1997) comentam que a presença de biomassa com boas características de sedimentação (lodo granular) é essencial para a operação do ASBR permitido alta eficiência de remoção de matéria orgânica e maior tempo de retenção celular.

De acordo com Sung e Dague (1995), reatores ASBR com diferentes características geométricas impõem diferentes níveis de "seleção" da biomassa durante cada ciclo total. Os autores verificarm que a utilização da relação de H/D entre 1,8 a 5,6 é preferível e mais favorável para a estabilidade do processo de tratamento, em vez da relação entre 0,6 a 0,9 , em razão da formação de biomassa com melhor característica de sedimentação. Nos reatores ASBR1 e ASBR3, não se pôde comprovar a implicação da característica geométrica no desempenho, mesmo utilizando inóculo granular. Houve perda de massa de lodo ao longo dos 70 dias de operação (Figura 4).

Os dados de concentração de lodo e quantidade de biomassa no interior dos reatores confirmam a perda ocorrida. Inicialmente, o lodo de inóculo, aplicado igualmente nos reatores, tinha as concentrações de 64,1 gST. $l^{-1}(25,6 \mathrm{kgST})$ e $52,8 \mathrm{gSV} . l^{-1}(21,1 \mathrm{kgSV})$, e, ao término de 70 dias, esses valores decresceram para 8,0 gST. $l^{-1}(3,3 \mathrm{kgST})$ e $6,90 \mathrm{gSV} . l^{-1}(2,8 \mathrm{kgSV})$ no ASBR1, enquanto no ASBR3 para $8,9 \mathrm{gST} . l^{-1}(3,7 \mathrm{kgST})$ e 7,3 gSV. $l^{-1}(3,0 \mathrm{kgSV})$. Portanto, a análise comparativa de desempenho entre as unidades, principalmente, com os reatores ASBR1 e ASBR3 que utilizam a recirculação de líquido como forma de agitação tornou-se inviável.

Ao contrário do ASBR1 e ASBR3, observou-se no ASBR2, que a perda de biomassa também ocorreu, mas devido, possivelmente, a seleção do lodo com melhor característica de sedimentação. Após 50 dias, estes valores decresceram no ASBR2 para 17,1 gST.l ${ }^{1}(7,0 \mathrm{kgST})$ e $14,1 \mathrm{gSV} . \mathrm{l}^{-1}(5,8 \mathrm{kgSV})$ e ao final atingiram $24,4 \mathrm{gST} . l^{-1}(10 \mathrm{kgST})$ e $21,2 \mathrm{gSV} . l^{-1}(8,7$ $\mathrm{kgSV})$. 
Tabela 5: Resultados do monitoramento dos reatores ASBR e ASBBR (20 amostras)

\begin{tabular}{|c|c|c|c|c|c|}
\hline \multirow{2}{*}{ Variável } & \multirow{2}{*}{ Afluente } & \multicolumn{4}{|c|}{ Efluente } \\
\hline & & ASBR1 & ASBR2 & ASBR3 & ASBBR \\
\hline Carga Orgânica (kgDQO. $\left.\mathrm{m}^{-3} \cdot \mathrm{d}^{-1}\right)$ & $0,6-1,2$ & - & - & - & - \\
\hline $\mathrm{DQO}_{\text {Bruta }}\left(\mathrm{mg} . l^{-1}\right)$ & $\begin{array}{c}563 \pm 118 \\
(800-345)\end{array}$ & $\begin{array}{c}334 \pm 69 \\
(452-215)\end{array}$ & $\begin{array}{c}215 \pm 50 \\
(374-134)\end{array}$ & $\begin{array}{c}314 \pm 70 \\
(517-214)\end{array}$ & $\begin{array}{c}215 \pm 50 \\
(356-148)\end{array}$ \\
\hline $\operatorname{DQO}_{\text {Filtrada }}\left(\mathrm{mg} . l^{-1}\right)$ & $\begin{array}{c}190 \pm 37 \\
(244-120)\end{array}$ & $\begin{array}{c}160 \pm 35 \\
(225-92)\end{array}$ & $\begin{array}{c}120 \pm 23 \\
(154-68)\end{array}$ & $\begin{array}{c}159 \pm 31 \\
(200-93)\end{array}$ & $\begin{array}{c}127 \pm 28 \\
(188-82)\end{array}$ \\
\hline *Eficiência de remoção ${ }_{\text {Bruta }}(\%)$ & (2) & $\begin{array}{c}39 \\
(59-11)\end{array}$ & $\begin{array}{c}60 \\
(75-32)\end{array}$ & $\begin{array}{c}43 \\
(63-16)\end{array}$ & $\begin{array}{c}61 \\
(72-46)\end{array}$ \\
\hline *Eficiência de remoção ${ }_{\text {Filtrada }}(\%)$ & - & $\begin{array}{c}71 \\
(83-45)\end{array}$ & $\begin{array}{c}78 \\
(89-70)\end{array}$ & $\begin{array}{c}71 \\
(85-60)\end{array}$ & $\begin{array}{c}77 \\
(87-66)\end{array}$ \\
\hline $\operatorname{AVT}\left(\operatorname{mgHac} . l^{-1}\right)$ & $\begin{array}{c}68 \pm 13 \\
(91-36)\end{array}$ & $\begin{array}{c}58 \pm 13 \\
(79-28)\end{array}$ & $\begin{array}{c}40 \pm 11 \\
(58-19)\end{array}$ & $\begin{array}{c}55 \pm 11 \\
(73-28)\end{array}$ & $\begin{array}{c}49 \pm 12 \\
(67-27)\end{array}$ \\
\hline $\mathrm{AB}\left(\mathrm{mgCaCO}_{3} \cdot l^{-1}\right)$ & $\begin{array}{c}84 \pm 16 \\
(109-40)\end{array}$ & $\begin{array}{c}106 \pm 21 \\
(163-73)\end{array}$ & $\begin{array}{c}133 \pm 24 \\
(215-101)\end{array}$ & $\begin{array}{c}111 \pm 16 \\
(155-86)\end{array}$ & $\begin{array}{c}118 \pm 20 \\
(157-88)\end{array}$ \\
\hline $\mathrm{pH}$ & $7,2-6,6$ & $7,1-6,8$ & $7,2-6,8$ & $7,1-6,9$ & $7,3-6,9$ \\
\hline $\mathrm{SST}\left(\mathrm{mg} . l^{-1}\right)$ & $\begin{array}{c}250 \pm 135 \\
(593-120)\end{array}$ & $\begin{array}{c}91 \pm 30 \\
(124-63)\end{array}$ & $\begin{array}{c}53 \pm 12 \\
(74-32)\end{array}$ & $\begin{array}{c}83 \pm 51 \\
(107-37)\end{array}$ & $\begin{array}{c}62 \pm 29 \\
(136-36)\end{array}$ \\
\hline $\operatorname{SSV}\left(\mathrm{mg} \cdot l^{-1}\right)$ & $\begin{array}{c}203 \pm 96 \\
(451-112) \\
\end{array}$ & $\begin{array}{c}81 \pm 26 \\
(115-52) \\
\end{array}$ & $\begin{array}{c}44 \pm 09 \\
(116-33)\end{array}$ & $\begin{array}{c}75 \pm 20 \\
(100-33)\end{array}$ & $\begin{array}{c}54 \pm 24 \\
(116-33)\end{array}$ \\
\hline *Remoção SST (\%) & - & 64 & 79 & 67 & 75 \\
\hline *Remoção SSV (\%) & - & 60 & 79 & 63 & 73 \\
\hline
\end{tabular}

Deve-se ressaltar que os dados de eficiência de remoção em termos de $\mathrm{DQO}_{\text {Bruta }}$ SST e SSV foram obtidos pela diferença entre a concentração média presente no afluente e do efluente dividido pela média do afluente nas 20 amostras analisadas. Apenas em

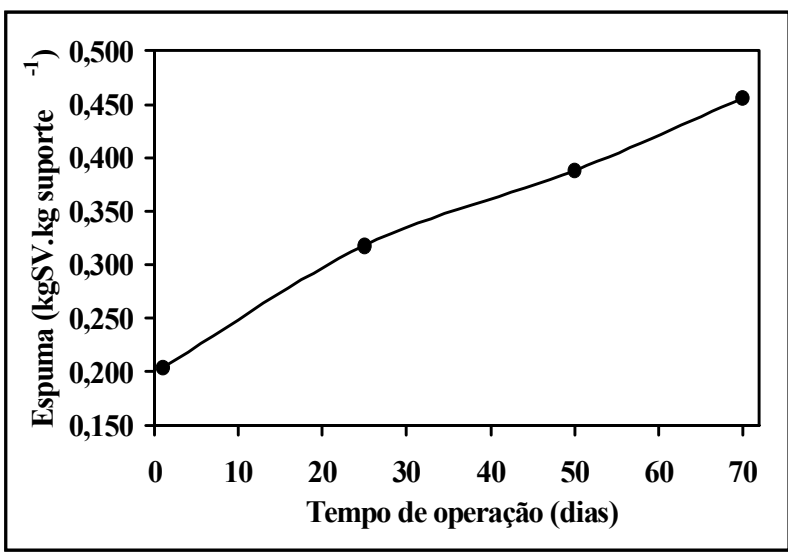

Figura 3. Concentração de sólidos voláteis na espuma de poliuretano ao longo de 70 dias de operação do reator ASBBR. termos de $\mathrm{DQO}_{\text {Filtrada }}$, a determinação de eficiência de remoção é realizada pela diferença de concentração média afluente bruta e a concentração no efluente filtrada dividida pela concentração média afluente bruta.

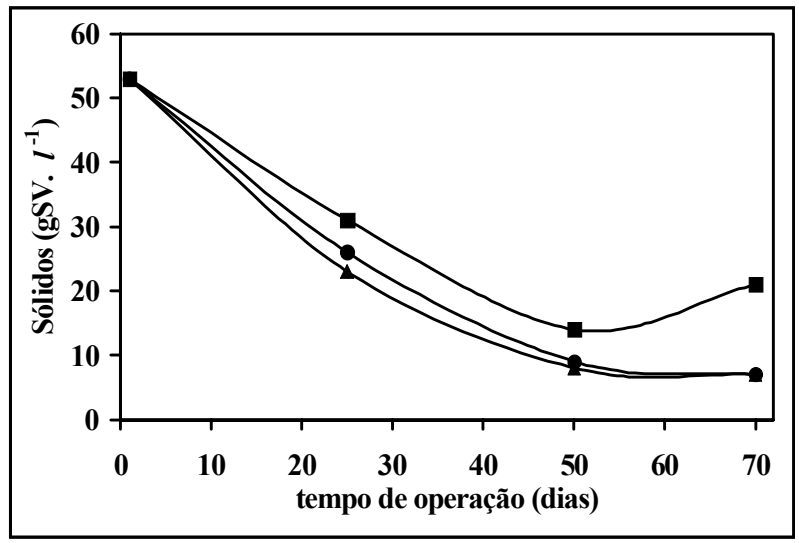

Figura 4. Concentração de sólidos voláteis do lodo ao longo de 70 dias de operação dos reatores ASBR1 (p), ASBR2 (ф) e ASBR3 (·). 
A agitação mecânica mostrou ser a mais indicada para utilização nos reatores ASBR, principalmente quando se comparou o desempenho entre o ASBR1, ASBR2 e o ASBR3. O ASBR2 demonstrou ser mais eficiente, e seu desempenho é comparável ao do ASBBR com meio suporte. Essa alternativa de agitação (mecânica) foi preconizada por Timur e Özturk (1999) e Ruiz et al. (2002) que utilizaram reatores ASBR, em escala de laboratório, com o uso de agitadores magnéticos. Bodik, Herdová e Drtil (2002) e Rodrigues et al. (2003) empregando sistema de agitação mecânica, formado de eixo e impelidor, verificaram também a viabilidade de uso deste tipo de agitação no ASBR.

Devido à presença de meio suporte no ASBBR, o efeito de remoção física de sólidos foi rapidamente sentida (Figura 3). Esse efeito ocorreu nos primeiros dias de operação, e refletiu nas eficiência de remoção de DQO e SST. Portanto, a imobilização em meio suporte, como a espuma de poliuretano oferece maior rapidez na retenção de sólidos em suspensão, colonização e adaptação da biomassa anaeróbia, e propicia curtos tempos de partida e operação mais estável. Esse sistema foi o que apresentou melhor eficiência no tratamento de esgoto sanitário, bem como na qualidade do efluente final. O comportamento do ASBBR foi semelhante ao ASBR2, conforme os resultados apresentados na Tabela 5.

No ASBBR a preocupação com a ocorrência do fenômeno de granulação nos reatores ASBR deixa de existir, de modo que é possível eliminar a fase de sedimentação, pois grande parte da biomassa está imobilizada no suporte inerte. A velocidade de agitação utilizada $40 \mathrm{rpm}$, conforme os trabalhos de Ratuzsnei et al. (2001), Pinho et al. (2002), Cubas et al. (2002) e Cubas et al. (2004), comprova que essa velocidade aplicada pode influenciar positivamente no desempenho do reator tipo ASBBR, bem como, o tipo de impelidor e tamanho dos cubos de espuma de poliuretano.

\section{Conclusões}

No estudo comparativo dos resultados de monitoramento na operação dos reatores ASBR1/ ASBR2 (diferente tipo de agitação e mesma relação H/D) e o ASBR1/ASBR3 (igual tipo de agitação e diferente relação H/D) foram observados baixos desempenhos no tratamento de esgoto sanitário nas unidades com recirculação de líquido, devido principalmente à baixa retenção de sólidos, influenciada pelo uso de bombas centrífugas. Nas unidades (ASBR1 e ASBR3), em termos de eficiência médias de remoção de DQO e SST, foram atingidas as faixas de 39 a $43 \%$ e de 60 a $67 \%$, respectivamente. A utilização de recirculação de líquido nos reatores do tipo ASBR precisa ser investigada tanto na sua concepção física como operacional (condições de agitação), a fim de que se possa atingir qualidade de efluente ideal no tratamento anaeróbio de esgoto sanitário.

Os melhores desempenhos foram apresentados pelos reatores ASBR2 e ASBBR. O ASBR2 demonstrou ser o mais eficiente entre os reatores do tipo ASBR, e seu desempenho foi comparável ao do ASBBR com meio suporte. Os resultados médios de eficiência de remoção de DQO e SST foram de $60 \%$ e $79 \%$ no ASBR2, enquanto no ASBBR de $61 \%$ e $75 \%$. Nesse caso, o uso de agitação mecânica mostrou ser a mais indicada para a operação destes tipos de reatores de fluxo descontínuo. Tais resultados demonstram o potencial dessas configurações de reatores anaeróbios no tratamento de esgoto sanitário, e elas podem ser mais uma opção para esta finalidade.

\section{Agradecimentos}

Os autores agradecem à Fundação de Amparo à Pesquisa do Estado de São Paulo - FAPESP (Processo: 99/06152-8) pelo suporte financeiro concedido. 


\section{Referências}

AMERICAN PUBLIC HEALTH ASSOCIATION. Standard methods for the examination of water and wastewater. 19 ed. Washington: American Public Health Association, 1995.

BODÍK, I.; HERDOVÁ, B.; DRTIL, M. The Use of Upflow Anaerobic Filter and AnSBR for Wastewater Treatment at Ambient Temperature. Water Research, New York, v.36, p.1084-1088, 2002.

CHERNICHARO, C. A. L.; VAN HAANDEL, A.; CAVALCANTI, P. F. F. Controle Operacional de Reatores Anaeróbios. In: CAMPOS, J. R.(Coord.). Tratamento de esgotos sanitários por processo anaeróbio e disposição controlada no solo. Rio de Janeiro: Associação Brasileira de Engenharia Sanitária e Ambiental, 1999. p. 221-247.

CUBAS, S. A.; PINHO, S. C.; FORESTI, E.; RODRIGUES, J. A. D.; RATUZSNEI, S. M.; ZAIAT, M. Effects of Solidphase Mass Transfer on a Stirred Anaerobic Sequencing Batch Reactor Containing Immobilized Biomes. In: SIMPÓSIO LATINOAMERICANO SOBRE DIGESTÃO ANAEROBIA, 7., 2002, Mérida. Anais... Yucatán, México, 2002. p.229-236.

CUBAS, S. A.; PINHO, S. C.; FORESTI, E.; RODRIGUES, J. A. D.; RATUZSNEI, S. M.; ZAIAT, M. Influence of the Liquid-phase Mass Transfer on the Performance of a Stirred Anaerobic Sequencing Batch Reactor Containing Immobilized Biomass. Biochemical Engineering Journal, Amsterdam, v.17, n.2, p.99-105, 2004.

DILALLO, R.; ALBERTSON, O. E. Volatile Acids by Direct Titration. Research Journal of the Water Pollution Control Federation, Alexandria, v.33, n.4, p. 356-365, 1961.

HENZE, M.; HARREMOES, P.; ARVIN, E.; JANSEN, J. C. Wastewater treatment: biological and chemical processes. New York: Springer, 1997.

HOLLOPETER, J. A; DAGUE, R. R. Anaerobic Sequencing Batch Reactor Treatment of Landfill Leachate. In: PURDUE INDUSTRIAL WASTE CONFERENCE , 49., 1994, Chelsea, Michigan. Proceedings... Chelsea, Michigan: Ann Arbor Press, 1994.

NDON, U. J.; DAGUE, R. R. Ambient Temperature Treatment of Low-Strength Wastewater Using Anaerobic Sequencing Batch Reactor. Biotechnology Letters, Dordrecht, v.19, n.4, p.319-323, 1997.

OLIVA, L. C. H. V. Tratamento de esgotos sanitários com reator anaeróbio de manta de lodo (UASB) protótipo: desempenho e respostas dinâmicas às sobrecargas hidráulicas. 1997. Tese (Doutorado) - Escola de Engenharia de São Carlos (EESC) - Universidade de São Paulo, São Carlos.
PINHO, S. C.; CUBAS, S. A.; RATUSZNEI, S. M.; RODRIGUES, J. A. D.; FORESTI, E.; ZAIAT, M. Influence of the Agitation Rate on the Treatment of Partially Soluble Wastewater in Anaerobic Sequencing Batch Reactor Containing Immobilized Biomass. In: SIMPÓSIO LATINO AMERICANO SOBRE DIGESTÃO ANAEROBIA, 7., Mérida, 2002. Anais... Yucatán, México, 2002. p.103-106.

RATUSZNEI, S. M.; RODRIGUES, J. A. D.; CAMARGO, E. F. M.; ZAIAT, M.; BORZANI, W. Feasibility of a Stirred Anaerobic Sequencing Batch Reactor Containing Immobilized Biomass for Wastewater Treatment. Bioresource Technology, Essex, Inglaterra, v.75, p.127-133, 2000.

RATUSZNEI, S. M.; RODRIGUES, J. A. D.; CAMARGO, E. F. M.; ZAIAT, M.; BORZANI, W. Influence of the Agitation Rate on the Performance of a Stirred Anaerobic Sequencing Batch Reactor Containing Immobilized Biomass. Water Science and Technology, Oxford, v.44, n.4, p.305-412, 2001.

RIBEIRO, R.; VARESCHE, M. B. A.; FORESTI, E.; ZAIAT, $M$. Influence of the Carbon Source on the Anaerobic Biomass Adhesion on Polyurethane Foam Matrices. Journal of Environmental Management, London, v.74, p.187-194, 2005.

RIPLEY, L. E; BOYLE, W. C; CONVERSE, J. C. Improved Alkalimetric Monitoring for Anaerobic Digestion of Highstrength Wastes. Research Journal of the Water Pollution Control Federation, Alexandria, v.58, n.5, p.406411, 1986.

RODRIGUES, J. A. D.; RATUSZNEI, S. M.; CAMARGO, E. F. M.; ZAIAT, M. Influence of the Agitation Rate on the Performance of a Anaerobic Sequencing Batch Reactor Containing Granulated Biomass Treating Low-Strength Wastewater. Advances in Environmental Research, Oxford, v.7, n. 2, p.405-410, 2003.

RUIZ, C.; TORIJOS, M.; SOUSBIE, P.; LEBRATO MARTINEZ, J.; MOLETTA, R.. The Anaerobic SBR Process: Basic Principles for Design and Automation. Water Science and Technology, Oxford, v.38, p.255-264, 2002.

SUNG, S.; DAGUE R. R. Laboratory Studies on the Anaerobic Sequencing Batch Reactor. Water Environmental Research, Alexandria, v.67, n.3, p.294-301, 1995.

TIMUR, H.; ÖZTURK, I. Anaerobic Sequencing Bath Reactor Treatment of Landfill Leachate. Water Research, New York, v.33, n. 15, p.3225-3230, 1999.

WIRTZ, R. A.; DAGUE, R. R. Enhancement of Granulation and Start-up in the Anaerobic Sequencing Batch Reactor. Water Environmental Research, Alexandria, v.68, n.5, p.883-892, 1996. 
